\title{
Aspect Based Emotion Detection and Topic Modeling on Social Media Reviews
}

\author{
Mr. Ganesh N. Jorvekar ${ }^{\mathrm{a}, 1}$ and Dr Mohit Gangwar ${ }^{\mathrm{b}}$ \\ ${ }^{a}$ Ph.D. Research Scholar, Department of Computer Science and Engineering, Bhabha University, Bhopal, \\ Madhya Pradesh, India \\ ${ }^{b}$ Dean-Engineering, Bhabha University, Bhopal, Madhya Pradesh, India
}

\begin{abstract}
In recent years, the number of user comments and text materials has increased dramatically. Analysis of the emotions has drawn interest from researchers. Earlier research in the field of artificial-intelligence concentrate on identification of emotion and exploring the explanation the emotions can't recognized or misrecognized. The association between the emotions leads to the understanding of emotion loss. In this Work we are trying to fill the gap between emotional recognition and emotional co-relation mining through social media reviews of natural language text. The association between emotions, represented as the emotional uncertainty and evolution, is mainly triggered by cognitive bias in the human emotion. Numerous types of features and Recurrent neural-network (RNN) as deep learning model provided to mine the emotion co-relation from emotion detection using text. The rule on conflict of emotions is derived on a symmetric basis. TF-IDF, NLP Features and Co-relation features has used for feature extraction as well as section and Recurrent Neural Network (RNN) and Hybrid deep learning algorithm for classification has used to demonstrates the entire research experiments. Finally evaluate the system performance with various existing system and show the effectiveness of proposed system.
\end{abstract}

Keywords- Aspect mining, text processing, feature extraction, feature selection, classification, machine learning, NLP

\section{Introduction}

On social media peoples share a lot of data in the form of text, photos, and videos in memes, whether personal, everyday scenes, or their opinions. The Internet is a massive forum for global and instantaneous communication and knowledge exchange, providing users with a decent selection of people's viewpoints and thoughts on a huge range of topics. There are rarely any textual captions in most social media posts, but they are filled with images. This largely leads to a spectrum of views and emotions being articulated quite implicitly only through visual content.

Wang Xinzhi et. Implemented Expression Connection Processing on Natural Source Language Via Deep Learning Models, which undermines the connection of emotions depending on the success of speech signals from state-of-the-art deep learning techniques.

\footnotetext{
${ }^{1}$ Mr. Ganesh N, Ph.D. Research Scholar, Department of Computer Science and Engineering, Bhabha University

Email.ganesh.jorvekar83@gmail.com.
} 
Some subjective individual by the dataset are expressed in the system and models are minimized by designing three kinds of features and two artificial learning models. Via an emotion misunderstanding law, which is arbitrary, and an expression evolution legislation, which is guided, the expression connection is mined. Studies are conducted on open to interpretation and long-short Online news messages. There are some promising emotional association studies

Via email, text or images, one may convey feelings. Although several works have used techniques to decode feelings from user posts in the past, especially on social media $[2,3]$, text sentiment analysis has yet to be explored. In the current situation, social media use to convey feelings is growing, so it is an important field of study. The new technologies are aimed at improving accuracy. For text sentiment analysis, there are several algorithms and techniques proposed.

We aim to find out if there are benefits to applying $\mathrm{CNN}$ to the study of visual emotions.

How to fine-tune training data identified on a limited scale distinct from the dataset to identify text, shall be explored. It may only be possible to perform learning in top layers during back propagation, as they have less specific data set features. The experimental findings show that this domain-specific fine-tuning for heterogeneous text classification effectively improves neural network performance.

\section{Literature Survey}

Xinzhi Wang et.al. (2020) [1], In the past few years, the proposal of emotion analysis has garnered a lot of attention among researchers. Many AI research papers deal with how to identify the feelings, but very few explore why it is that anyone might mistake his or her own for anything else. The fact that the association among emotions contributes to the problems associated with the inability to recognise them. in this study, we are working to bridge the gap between emotional sentiment identification and traditional content analyses by providing more data from articles harvested from the Web. Humans often express their emotions as the tendency to change in emotional expressions, thereby creating the confused and evolving nature of those expressions. Emotion is detected by three kinds of features and two deep neural network models, two of which are deployed in tandem to expand on their meaning. It's estimated that people experience two thousand types of emotions in a movie. From three viewpoints, the hypothesis of emotion evolution is supported. 1) change only happens in one direction at a time, 2) in small steps, and 3) on the shortest path The technique is rigorously examined in three investigations. 1) title examples; 2) and news article body examples of statements spanning both positive and negative terms; and negative content ranging from short ones to long-form texts (long and short). The experimental evidence indicates that people quickly misinterpret emotional statements when they are unaccompanied by other cues. When people are emotionally touched by comments, such feelings appear to create feelings of love or rage. It is obvious in print whether fear or joy is being portrayed; in print, it is simple to identify the emotion of fear or joy being recorded. According to the study's results, some applications, such as affective interactions, social media and public opinion analysis, as well as well as humancomputer interaction, this could be very helpful. 
Renata L. Rosa et.al. (2019) [2], The author argued that social networks (e.g., OSNs) ought to include information about various user-related topics that was pertinent to the individuals' lives. This application gathers and analyses this type of data while allowing it to track and suggest other applications, such as application monitoring and application recommendations. In this paper, we describe a Knowledge-Based System (KBS), which involves a mental wellbeing monitoring to find those who may be suffering from psychological issues, in particular. Depending on the outcome of the monitoring, one's findings, we could send messages that assist users suffering from anxiety, for better understanding of concepts, the system will rely on KBRS, KB knowledge, and sentiment analysis. To ensure long-term success, there is a feature to alert trusted users in the event of any deviation from the set parameters is built in, particularly with regards to potential itself with building models that identify the cause and effect relationships between different items of data. However, the observation that online social networks tend to expand a user's ability to make connections could mean they could result in a decreased amount of actual face-to-to-face interactions. A massive outbreak is the result of these phenomena, and these people now have the words such as Phubbing (This word, according to my opinion, is about laziness) and Nomophobia (This word, for me, denotes fear of being out of cell phones) to identify them.

\section{Proposed System Design}

The proposed research paper on text sentiment classification uses a strong machine learning technique called deep learning Based on the examples given, the application illustrates numerous text extraction and data building options and principles are added to the train's understanding. Feature extraction techniques including aspects like form, texture, alpha density, and colour have been implemented in order to derive other attributes such as amplitude and tone. Most times, it is used to define text meta-data to include categorization of thoughts as well. In order to attain higher classification accuracy, normalizing the data has the data has the biggest influence.

Features have been taken from the training data collection and applied during training, and a training model has been developed accordingly. The model that allows each textual function to be introduced with the same amount of strength was added to the testing results. the weight estimation approach takes into account the attributes of research and preparation, as well as one other related attributes It's a part of the subprocess of describing the similarities between two sets of characteristics. by using weighting factors that build on the factor in their appetite, the factors according to their respective emotional responses A perfect weight is completely subjective; it is entirely up to the customer to the individual to decide. You will find more detail here.

1. Text resizing. Evaluate each text's height and width accordingly and adjust it to the appropriate size.

2. Often text contains some noise or a certain kind of noise already contains unique input text. We remove noise from images using the Gaussian noise filter. 


\section{Results and Discussions}

The To measure the accuracy for the method, the matrix has to be expanded a multitier java 3. 2.2 GHz and 512 MB RAM on the implementation using the OPENstep Java Runtime Environment; we have made it the calls to the web server using 1.3MB and $2 \mathrm{MB}$ of Java SE class directories. After the evaluation of various systems and alternatives has been done, the preference of a more sophisticated system could have been reached.

This experiment we analyse the classification accuracy of ReLU using twitter dataset, the similar experiments has done with various cross validation and results has illustrates in table 1 . According to this analysis we conclude 10 -fold cross validation provides highest $95.30 \%$ and $96.10 \%$ for 10 -fold cross validation classification accuracy for RNN.

Table 1. Average classification accuracy with confusion matrix RNN

\begin{tabular}{|l|l|l|l|}
\hline RNN & Fold 5 & Fold 10 & Fold 15 \\
\hline Accuracy & 94.20 & 95.30 & 96.10 \\
\hline Precision & 94.30 & 95.70 & 95.30 \\
\hline Recall & 94.15 & 95.80 & 96.40 \\
\hline F1 Score & 93.20 & 95.60 & 96.50 \\
\hline
\end{tabular}

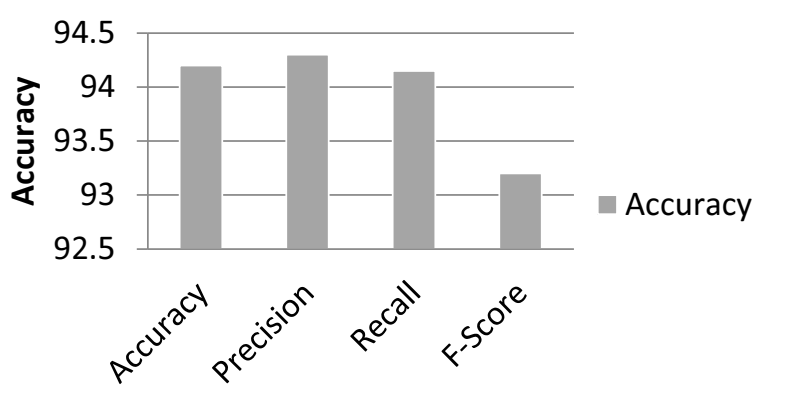

Figure 1. System classification accuracy using RNN with 5-fold data cross validation

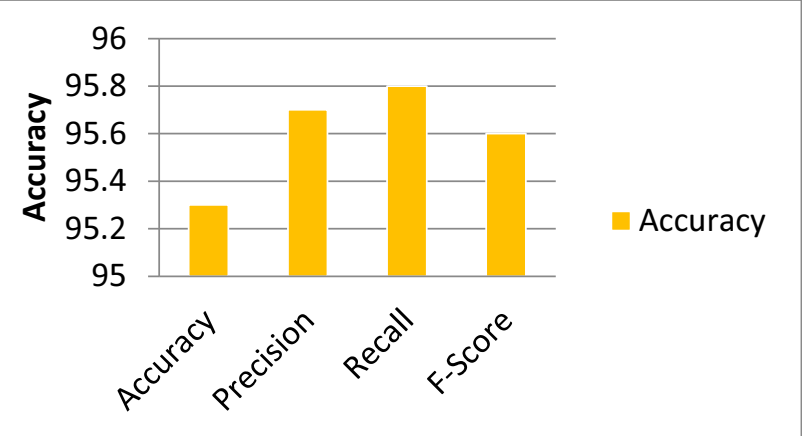

Figure 2. System classification accuracy using RNN with 10 -fold data cross validation 


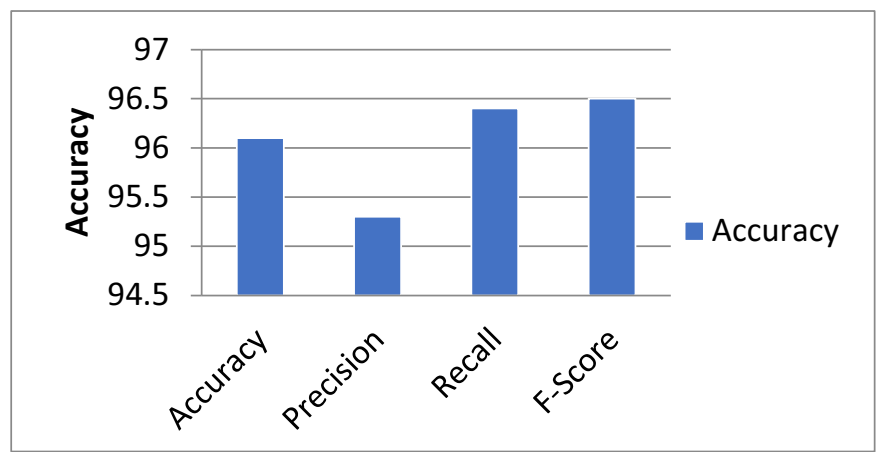

Figure 3. System classification accuracy using RNN with 15 -fold data cross validation

\section{Conclusions}

Our research examines the techniques of deep learning in text sentiment classification schemes. in programmatic classification, videos provide several representations of all three of the three of these emotions in a row in a specific order, thereby categorising them as thrillers, romantics, and comedy, with about a third being labelled as action. According to the survey, it was found that there are three different types of RNN (overregularization, under-regularization, and over-regularization) as the method works on different datasets. can be expressed as a total state expansion of the following DNN utilising more than $96 \%$ of the available Deep4 resources, making it the most efficient. Our observational study indicates that, along with the classification of emotions, the Deep Learning algorithm provides promising text data results. The future study involves conducting tests in a manufacturing environment on a wide scale. In addition to pictures, video scenes can be accommodated to further categories feelings into various genres such as happy, thriller, humorous, romance etc.

\section{References}

[1]. Xinzhi Wang, Luyao Kou, Vijayan Sugumaran, Xiangfeng Luo, and Hui Zhang, "Emotion Correlation Mining Through Deep Learning Models on Natural Language Text", IEEE TRANSACTIONS ON CYBERNETICS, May 2020.

[2]. Renata L. Rosa, Gisele M. Schwartz, Wilson V. Ruggiero, and Demostenes Z. Rodr 1guez, Senior Member, IEEE, "A Knowledge-Based Recommendation System that includes Sentiment Analysis and Deep Learning", IEEE Transactions on Industrial Informatics Vol. 15, April 2019.

[3]. B. Liu,"Many facets of sentiment analysis, a practical guide to sentiment analysis," Springer International Publishing, pp. 11-39,Jan 2017.

I. B. Weiner and R. L. Greene, "Handbook of personality assessment," in John Wiley and Sons, N.J, EUA, 2008.

[4]. H. Lin, J. Jia, J. Qiu, Y. Zhang, G. Shen, L. Xie, J. Tang, L. Feng, and T. S. Chua, "Detecting stress based on social interactions in social networks," IEEE Transactions on Knowledge and Data Engineering, vol. 29, no. 9, pp. 18201833, Sept 2017. 
[5]. J. T. Hancock, K. Gee, K. Ciaccio, and J. M.-H. Lin, "I'm sad you're sad. Emotional contagion in cmc," in Proceedings of the 2008 ACM Conference on Computer Supported Cooperative Work, pp. 295-298, Year 2008.

[6]. Afzaal M, Usman M and Fong A 2019 "Tourism Mobile App with AspectBased Sentiment Classification Framework for Tourist Reviews", IEEE Transactions on Consumer Electronics (IEEE). 65. 233-242. doi.10.1109/TCE.2019.2908944.

[7]. Rana T A, Cheah Y N 2017 "Improving aspect extraction using aspect frequency and semantic similarity-based approach for aspect-based sentiment analysis", In. International Conference on Computing and Information Technology, Springer, Cham, doi.10.1007/978-3-319-60663-7_30, pp. 317-326.

[8]. I.-R. Glavan, A. Mirica, and B. Firtescu, "The use of social media for communication.”, Official Statistics at European Level. Romanian Statistical Review, vol. 4, pp. 37-48. Dec. 2016,.

[9]. M. Al-Qurishi, M. S. Hossain, M. Alrubaian, S. M. M. Rahman, and A. Alamri, "Leveraging analysis of user behavior to identify malicious activities in largescale social networks," IEEE Transactions on Industrial Informatics, vol. 14, no. 2, pp. 799-813, Feb 2018.

[10]. R. L. Rosa, D. Z. Rodr' 1guez, and G. Bressan, "Music recommendation system based on user's sentiments extracted from social networks," IEEE Transactions on Consumer Electronics, vol. 61, no. 3, pp. 359-367, Oct 2015.

[11]. R. Rosa, D. Rodr, G. Schwartz, I. de Campos Ribeiro, G. Bressan et al., "Monitoring system for potential users with depression using sentiment analysis," in 2016 IEEE International Conference on Consumer Electronics (ICCE). Sao Paulo, Brazil. IEEE, pp. 381-382, Jan 2016.

[12]. Y. P. Huang, T. Goh, and C. L. Liew, "Hunting suicide notes in web 2.0 preliminary findings," in Ninth IEEE International Symposium on Multimedia Workshops (ISMW 2007), pp. 517-521. Dec 2007.

[13]. W. H. Organization, "World health statistics 2016. Monitoring health for the sustainable development goals, world health statistics annual," World Health Organization, p. 161, 2016.

[14]. Hu M, Liu B 2004, "Mining and summarizing customer reviews.", In. Proceedings of the tenth ACM SIGKDD international conference on Knowledge discovery and data mining, doi.10.1145/1014052.1014073, pp. 168-177. 